\title{
NONLINEAR MONOTONIZATION OF THE BABENKO SCHEME FOR THE QUASI-LINEAR ADVECTION EQUATION ${ }^{1}$
}

\author{
T.A. ALEXANDRIKOVA and M.P. GALANIN
}

Keldysh Institute of Applied Mathematics of RAS

Miusskaya Sq. 4, Moscow A-47, 125047, Russia

E-mail: tatianaaleks@inbox.ru; galan@keldysh.ru

Received October 21, 2004; revised March 4, 2005

\begin{abstract}
The paper is devoted to construction and development of new method for numerical solution of hyperbolic type equations $[14,17]$.

In the previous papers $[4,5,6,7,8,9]$ authors have investigated theoretically and tested experimentally 26 different finite-difference schemes on 4 point patterns for the simplest hyperbolic equation: linear advection equation. This equation has the main features of every hyperbolic equation and is the important part of many mathematical models. In other cases the advection operator is the important part of the full operator of the problem. All 26 schemes have been compared experimentally on the special representative set of tests. Nevertheless to simplicity of the equation, almost all schemes have different disadvantages. They are discussed in detail in the cited papers. So, the investigation of new schemes for this equation is still an important task.

In $[4,5,6,7,8,9]$ some new schemes were constructed for solving this advection equation. The nonlinear monotone Babenko scheme ("square") proved to be the best among all 26 schemes. So, it is a big interest to generalize this scheme to more difficult equations. The important example is a quasi-linear advection equation.

In this paper our basic aim is to construct a quasi-monotone nonlinear Babenko scheme for solving the quasi-linear advection equation and to test it experimentally. The monotonisation of the scheme is done by adding the artificial diffusion with limiters. We also present advanced results of comparative analysis of the new scheme with other known schemes. We have considered explicit and implicit upwind approximation schemes $[4,6,13,16]$ which is firstorder accurate in time and space, the Lax-Wendroff scheme [4] which is the first order accurate in time and second order accurate in space. We also analyze the monotonised "Cabaret" scheme proposed in $[10,11]$. It is second order accurate in time and space, and its monotonisation is based on apriori knowledge of the dependence region of the exact solution. The authors of this
\end{abstract}

\footnotetext{
${ }^{1}$ Authors are grateful to T.G. Yelenina for her help and useful discussions. This research was partially supported by the Russian Foundation for Basic Research (RFBR project 03-0100461).
} 
scheme called it by "jumping advection". The considered schemes are compared numerically by using a set of tests, which is similar to one used in $[4,5,6,8]$.

Key words: Nonlinear Babenko scheme, quasi-linear advection equation

\section{Problem statement}

Let us consider the Cauchy problem for the quasi-linear advection equation

$$
\frac{\partial u}{\partial t}+\frac{\partial}{\partial x}\left(\frac{u^{2}}{2}\right)=0, \quad x \in(-\infty,+\infty), \quad t \in(0, T]
$$

with fi nite (mostly) initial condition $u(x, 0)=u_{0}(x)$. We are going to construct the Babenko scheme for this equation and to test it on the special set of test solutions. The initial functions for this set have following forms:

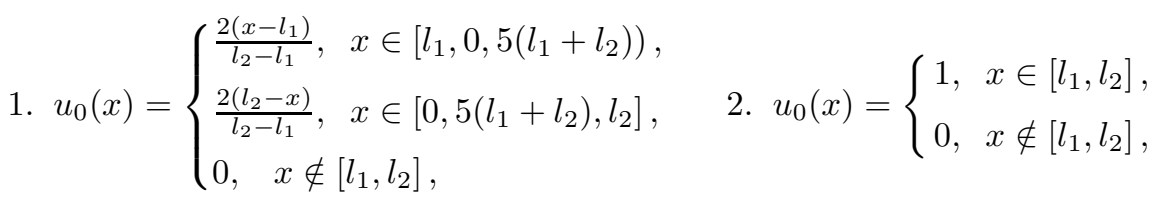

3. $u_{0}(x)=\left\{\begin{array}{l}\frac{\left(x-l_{1}\right)}{l_{2}-l_{1}}, x \in\left[l_{1}, l_{2}\right], \\ 0, x \notin\left[l_{1}, l_{2}\right],\end{array} \quad\right.$ 4. $u_{0}(x)=\left\{\begin{array}{l}\frac{\left(l_{2}-x\right)}{l_{2}-l_{1}}, x \in\left[l_{1}, l_{2}\right] \\ 0, x \notin\left[l_{1}, l_{2}\right]\end{array}\right.$

5. $u_{0}(x)=\left\{\begin{array}{ll}1, & x \in\left(-\infty, l_{1}\right], \\ 0, & x \in\left[l_{1},+\infty\right),\end{array} \quad\right.$ 6. $u_{0}(x)= \begin{cases}0, & x \in\left(-\infty, l_{1}\right] \\ 1, & x \in\left[l_{1},+\infty\right)\end{cases}$

They have a form of triangle, rectangle, left-hand triangle, right-hand triangle, stepdown and step-up functions, respectively.

Here are the exact solutions for initial conditions defi ned above:

1.

$$
u(x, t)=\left\{\begin{array}{l}
\frac{2\left(x-l_{1}\right)}{l_{2}-l_{1}+2 t}, \quad x \in\left[l_{1}, 0.5\left(l_{2}+l_{1}\right)+t\right], 0<t<0.5\left(l_{2}-l_{1}\right), \\
\frac{2\left(l_{2}-x\right)}{l_{2}-l_{1}-2 t}, \quad x \in\left[0.5\left(l_{2}+l_{1}\right)+t, l_{2}\right], 0<t<0.5\left(l_{2}-l_{1}\right), \\
0, \quad x \notin\left[l_{1}, l_{2}\right], 0<t<0.5\left(l_{2}-l_{1}\right), \\
\frac{2\left(x-l_{1}\right)}{l_{2}-l_{1}+2 t}, x \in\left[l_{1}, l_{1}+\sqrt{\frac{1}{2}\left(l_{2}-l_{1}\right)\left(l_{2}-l_{1}+2 t\right)}\right], t \geq \frac{1}{2}\left(l_{2}-l_{1}\right), \\
0, \quad x \notin\left[l_{1}, l_{1}+\sqrt{0.5\left(l_{2}-l_{1}\right)\left(l_{2}-l_{1}+2 t\right)}\right], t \geq 0.5\left(l_{2}-l_{1}\right),
\end{array}\right.
$$


2.

$$
u(x, t)=\left\{\begin{array}{l}
\frac{x-l_{1}}{t}, \quad x \in\left[l_{1}, l_{1}+t\right], 0<t<2\left(l_{2}-l_{1}\right), \\
1, \quad x \in\left[l_{1}+t, l_{2}+0.5 t\right], 0<t<2\left(l_{2}-l_{1}\right), \\
0, \quad x \notin\left[l_{1}, l_{2}+0.5 t\right], 0<t<2\left(l_{2}-l_{1}\right), \\
\frac{x-l_{1}}{t}, \quad x \in\left[l_{1}, l_{1}+\sqrt{2\left(l_{2}-l_{1}\right) t}\right], t \geq 2\left(l_{2}-l_{1}\right), \\
0, \quad x \notin\left[l_{1}, l_{1}+\sqrt{2\left(l_{2}-l_{1}\right) t}\right], t \geq 2\left(l_{2}-l_{1}\right),
\end{array}\right.
$$

3.

$$
u(x, t)=\left\{\begin{array}{l}
\frac{x-l_{1}}{t+\left(l_{2}-l_{1}\right)}, \quad x \in\left[l_{1}, l_{1}+\sqrt{\left(l_{2}-l_{1}\right)\left(t+\left(l_{2}-l_{1}\right)\right)}\right] \\
0, \quad x \notin\left[l_{1}, l_{1}+\sqrt{\left(l_{2}-l_{1}\right)\left(t+\left(l_{2}-l_{1}\right)\right)}\right], \quad t \geq 0,
\end{array}\right.
$$

4.

$$
u(x, t)=\left\{\begin{array}{l}
\frac{x-l_{1}}{t}, \quad x \in\left[l_{1}, l_{1}+t\right], 0<t \leq\left(l_{2}-l_{1}\right), \\
\frac{l_{2}-x}{l_{2}-l_{1}-t}, \quad x \in\left[l_{1}+t, l_{2}\right], 0<t \leq\left(l_{2}-l_{1}\right), \\
0, \quad x \notin\left[l_{1}, l_{2}\right], 0<t \leq\left(l_{2}-l_{1}\right), \\
\frac{x-l_{1}}{t}, \quad x \in\left[l_{1}, l_{1}+\sqrt{\left(l_{2}-l_{1}\right) t}\right], t>\left(l_{2}-l_{1}\right), \\
0, \quad x \notin\left[l_{1}, l_{1}+\sqrt{\left(l_{2}-l_{1}\right) t}\right], t>\left(l_{2}-l_{1}\right),
\end{array}\right.
$$

5. $u(x, t)=\left\{\begin{array}{ll}1, & x \in\left(-\infty, l_{1}+0.5 t\right], \\ 0, & x \notin\left(-\infty, l_{1}+0.5 t\right] ;\end{array} \quad\right.$. $\quad u(x, t)=\left\{\begin{array}{l}0, x \in\left(-\infty, l_{1}\right), \\ \frac{x-l_{1}}{t}, x \in\left[l_{1}, l_{1}+t\right], \\ 1, x \in\left(l_{1}+t,+\infty\right) .\end{array}\right.$

To compare numerical and exact solutions we use fi nite-dimensional analogs of norms in spaces $C, L_{1}, L_{2}$.

\section{Nonlinear Monotonisation of the Babenko Scheme}

For the numerical solution let us introduce the uniform (for simplicity) space-time $\operatorname{grid} \bar{\omega}_{h \tau}=\bar{\omega}_{h} \times \bar{\omega}_{\tau}$ :

$$
\bar{\omega}_{\tau}=\left\{t_{n}=n \tau, \quad n=0,1,2 \ldots\right\}, \quad \bar{\omega}_{h}=\left\{x_{i}=i h, \quad i=0,1,2 \ldots\right\},
$$

where $h$ is the space grid step and $\tau$ is the time grid step.

Further we use the following standard notation of grid functions and operators $[15,16]$ 


$$
y=y_{i}^{n}, \hat{y}=y_{i}^{n+1}, \quad y_{+1}=y_{i+1}^{n}, \quad y_{-1}=y_{i-1}^{n}, \quad y_{t}=\frac{\hat{y}-y}{\tau}, \quad y_{\bar{x}}=\frac{y-y_{-1}}{h},
$$

where the superscript is a time layer number and the subscript is a number of point on $x$. We assume that the solution $y^{n}$ is known.

For equation (1.1) the Babenko scheme has the following form:

$$
\frac{1}{2} y_{t}+\frac{1}{2} y_{t,-1}+\frac{1}{4}\left(\hat{y}^{2}\right)_{\bar{x}}+\frac{1}{4}\left(y^{2}\right)_{\bar{x}}=0 .
$$

It can be rewritten as

$$
y_{t}+\frac{1}{2}\left(y_{t,-1}-y_{t}\right)+\frac{1}{2}\left(y^{2}\right)_{\bar{x}}+\frac{1}{4}\left[\left(\hat{y}^{2}\right)_{\bar{x}}-\left(y^{2}\right)_{\bar{x}}\right]=0 .
$$

Suppose $a=\frac{1}{2}(\hat{y}+y)$, then equation (2.1) takes the form:

$$
y_{t}+\frac{1}{2}\left(y_{t,-1}-y_{t}\right)+\frac{1}{2}\left(y^{2}\right)_{\bar{x}}+\frac{1}{2 h}\left[a \tau y_{t}-a_{-1} \tau y_{t,-1}\right]=0,
$$

or

$$
y_{t}+\frac{1}{2}\left(y^{2}\right)_{\bar{x}}+\frac{1}{2} y_{t,-1}\left(1-\gamma_{-1}\right)-\frac{1}{2} y_{t}(1-\gamma)=0,
$$

where $\gamma=a \tau / h$ is the Courant number. In the rest of the paper only difference derivatives are used. Therefore we use the notations for difference derivatives similar to the partial ones.

It is well known [5], that for smooth solutions the Babenko scheme [2, 3] is second order accurate in $x$ and $t$, but it is non-monotone. This is an implicit scheme, but it does not prevent us from calculating the solution on a next time layer by an explicit formula. For the linear advection equation the Babenko scheme gives the exact solution on some grids, when the corresponding Courant number is equal to 1 . Let us notice that fi rst two terms in (2.2) give the usual upwind scheme.

We monotonize the scheme by adding to (2.2) two terms

$$
-\frac{1}{2} \mu_{-1} y_{t,-1}\left(1-\gamma_{-1}\right), \quad \frac{1}{2} \mu y_{t}(1-\gamma),
$$

where $\mu$ is the artifi cial diffusion. Since time derivatives $u_{t}, u_{t,-1}$ of the exact solution approximate space derivatives $-\left(\frac{u^{2}}{2}\right)_{x},-\left(\frac{u^{2}}{2}\right)_{x,-1}$, the introduced items produce diffusive terms. Hence the modifi ed scheme takes the following form:

$$
y_{t}+\frac{1}{2}\left(y^{2}\right)_{\bar{x}}+\frac{1}{2} y_{t,-1}\left(1-\gamma_{-1}\right)\left(1-\mu_{-1}\right)-\frac{1}{2} y_{t}(1-\gamma)(1-\mu)=0 .
$$

Let us note that for $\mu \equiv 0$ we get the original Babenko scheme and for $\mu \equiv 1$ we get the upwind scheme.

We now rewrite (2.3) in the following form:

$$
y_{t}+\varphi\left(\frac{y^{2}}{2}\right)_{\bar{x}}=0
$$


where

$$
\varphi=\frac{1+\frac{1}{2}\left(1-\gamma_{-1}\right)\left(1-\mu_{-1}\right) y_{t,-1} /\left(y^{2} / 2\right)_{\bar{x}}}{1-\frac{1}{2}(1-\gamma)(1-\mu)}
$$

The scheme (2.3) is constructed taking into account the assumption that $y \geq 0$ in the whole space-time region. Then it satisfi es the maximum principle [15] if the following conditions are valid

$$
\left\{\begin{array}{l}
\varphi \geq 0 \\
1-\gamma \varphi \geq 0
\end{array}\right.
$$

Notice that quantity $\tilde{a}=\frac{1}{2}\left(y+y_{-1}\right)$ is used in the literal condition of maximum principle fulfi llment. So, it is different from the second inequality in (2.4). But the desirable condition follows from the second inequality in (2.4) if $\hat{y} \geq 0$. Let us introduce functions

$$
R_{-1}=\frac{y_{\bar{x}}^{2}}{2 y_{t,-1}}=\frac{\tau}{h} \frac{y^{2}-y_{-1}^{2}}{2\left(\hat{y}_{-1}-y_{-1}\right)}, \quad R=\frac{\tau}{h} \frac{y_{+1}^{2}-y^{2}}{2(\hat{y}-y)}
$$

and denote $\mu=\mu(R, \gamma), \mu_{-1}=\mu\left(R_{-1}, \gamma_{-1}\right)$.

Inequalities (2.4) are valid if function $\mu=\mu(R, \gamma)$ takes the form [5]:

$$
\mu(R, \gamma)=\left\{\begin{array}{l}
1, \quad R \geq 0, \\
1+\frac{2 R}{1-\gamma}, \quad-0.5(1-\gamma) \leq R<0, \\
0,-R^{*} \leq R \leq-0.5(1-\gamma), \\
\frac{2\left(R+R^{*}\right)}{1-\gamma},-R^{*}-0.5(1-\gamma) \leq R \leq-R^{*}, \\
-1, \quad R<-R^{*}-0.5(1-\gamma) .
\end{array}\right.
$$

Function $\mu$ provides the monotonisation of the considered scheme. Note that the third line in (2.6) corresponds to the line segment, where the scheme preserves the second order of approximation for linear and closed to linear profi les $[18,12]$. The fourth line in (2.6) provides change from zero to negative values of $\mu$. In this case anti-diffusion items in the scheme appear. They decrease the numerical diffusion at discontinuity points of the solution.

In this work parameter $R^{*}$ is selected experimentally and $R^{*}=1.2$ is used in all numerical experiments [5].

Equation (2.3) is nonlinear due to dependence of $R$ on $\hat{y}$. The unknown $\hat{y}$ value can be calculated using the known value of $R$ by:

$$
\hat{y}=y+\frac{\tau}{2 h} \frac{y_{+1}^{2}-y^{2}}{R} .
$$

We can find $R$ using relation $\mu=\mu(R, \gamma)$. First we rewrite equation (2.3) in the form:

$$
\begin{aligned}
(\hat{y}-y)(1- & 0.5(1-\gamma)(1-\mu)) \\
& +0.5\left(1-\gamma_{-1}\right)\left(1-\mu_{-1}\right)\left(\hat{y}_{-1}-y_{-1}\right)+\frac{\tau}{2 h}\left(y^{2}-y_{-1}^{2}\right)=0
\end{aligned}
$$


According to (2.5) this equation takes the following form:

$$
\begin{aligned}
\frac{1}{R}(1-0.5(1-\gamma)(1-\mu)) & \\
& =-\frac{0.5\left(1-\gamma_{-1}\right)\left(1-\mu_{-1}\right)\left(\hat{y}_{-1}-y_{-1}\right)+\frac{\tau}{2 h}\left(y^{2}-y_{-1}^{2}\right)}{\frac{\tau}{2 h}\left(y_{+1}^{2}-y^{2}\right)}
\end{aligned}
$$

We denote by $b$ the right-hand side of equation (2.9):

$$
b=-\frac{0.5\left(1-\gamma_{-1}\right)\left(1-\mu_{-1}\right)\left(\hat{y}_{-1}-y_{-1}\right)+\frac{\tau}{2 h}\left(y^{2}-y_{-1}^{2}\right)}{\frac{\tau}{2 h}\left(y_{+1}^{2}-y^{2}\right)} .
$$

Equation (2.9) with a known right-hand side is a nonlinear equation with respect to $R$. The left-hand side is a monotone function of $R$. Considering the form of function $\mu=\mu(R, \gamma)$, we get the following relations:

$$
R=\left\{\begin{array}{l}
\frac{1}{b}, \quad b>0, \\
\frac{\gamma}{b}, \quad \frac{\gamma}{-R^{*}-0.5(1-\gamma)}<b<0, \\
\frac{R^{*}+0.5(1+\gamma)}{b-1}, \quad-\frac{0,5(1+\gamma)}{R^{*}}<b \leq \frac{\gamma}{-R^{*}-0.5(1-\gamma)}, \\
\frac{0.5(1+\gamma)}{b}, \quad-\frac{1+\gamma}{1-\gamma}<b \leq-\frac{0.5(1+\gamma)}{R^{*}}, \\
\frac{1}{b-1}, \quad b \leq-\frac{1+\gamma}{1-\gamma} .
\end{array}\right.
$$

Remark 1. The value of $b$ is indefi nite if $y_{+1}^{2}-y^{2}=0$, but in this case $R=0$ (as it follows from (2.5)). Also, we get from (2.11) that the value of $R$ is indefi nite, if $b=0$, but in this case the equality $\hat{y}=y$ can be obtained directly from (2.10) and (2.8).

So, using equations (2.7), (2.10), (2.11) we can find $\widehat{y}$. Note that $\gamma$ depends on $\hat{y}$, therefore the value of $\hat{y}$ is calculated numerically using iteration method. In this work at fi rst iteration step we count the value of $y$ as $0.5\left(\widehat{y}_{-1}+y_{+1}\right)$. For this value we calculate $\gamma$, then we defi ne R basing on the known value of $b$ and calculate desired value of $\bar{y}$ using (2.7).

\section{Numerical Experiments}

As it was done in $[4,5,8]$ the numerical solution of problem (1.1) is obtained for all stated above initial data for following parameters:

$$
l=520, \quad l_{1}=0, \quad l_{2}=20, \quad T=1000, \quad h=1 .
$$

The Courant number $\gamma=\max _{x, t} \frac{\tau}{h}|y(x, t)|$ is bounded by $\gamma_{m}$, which took values $0.1,0.25,0.5,0.9$. For the implicit upwind scheme calculations were also made for $\gamma_{m}=3$. So, the time step $\tau$ took the same values as the Courant number. Results of numerical experiments are presented in [1]. 
To illustrate the features of different schemes we present below numerical solutions captured at some intermediate time moments. These solutions correspond to pointed above initial data. Every fi gure plots the exact solution and numerical solutions for different values of Courant number. Figures are presented for the new quasi-monotone Babenko scheme, explicit upwind scheme and jumping advection method. Specifi cation of results obtained for all considered schemes is also presented below.

The obtained results make possible to analyze qualitative and quantitative features of the new developed scheme and to compare it with the other schemes considered in this work.

\subsection{Explicit upwind scheme}

The solution obtained by this method is very rough, diffusion of solution discontinuity takes place at few steps (from 4 to 10 steps). If $\gamma$ is smaller, the solution diffusion is greater. The solutions of depression wave type are diffused on more steps compared with shock waves. This solution drawback grows in time. For initial profi les 1, 2,4 on time intervals where the exact solution evolutes without amplitude change the amplitude of numerical solution diminishes permanently. It is diffi cult to trace time moments when shock waves arise. The speed of propagation of shock waves for the numerical solution is the same as for exact solution and is independent of $\gamma$.

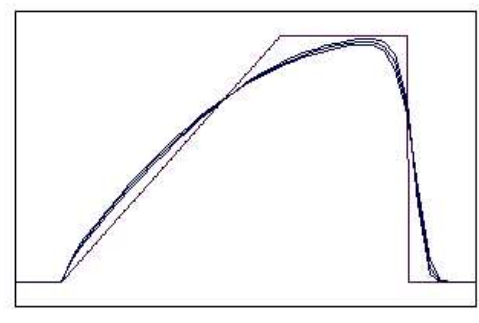

$t=6$

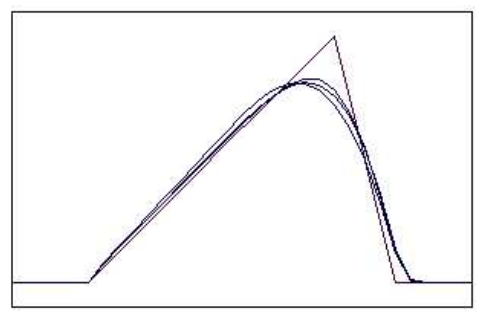

$t=10$

Figure 1. Numerical solution obtained using the implicit upwind scheme for 1 initial data $(\gamma=0.1 ; 0.5 ; 0.9)$.

\subsection{Implicit upwind scheme}

The solution obtained using this scheme is even less similar to exact solution than the solution obtained by using the previous one. For this scheme even greater solution diffusion is typical (from 7 to 15 space steps). This diffusion becomes stronger as the Courant number value increases. As shown in Figures 1-6, at initial time moments the bending of back front of the wave is typical for the numerical solution. In process of time the dependence of numerical solution on Courant number becomes less expressed. For initial profi les 1, 2, 3 and 4 the numerical solution obtained by using explicit and implicit upwind schemes evolutes to a wave of triangle profi le 
with diminishing amplitude and shock wave spreading to the right side. It conforms to the exact solution, but the maximum of numerical solution amplitude goes behind the maximum of the exact solution. So, the upwind schemes badly reproduce such features of solution as appearance and propagation of shock and depression waves.

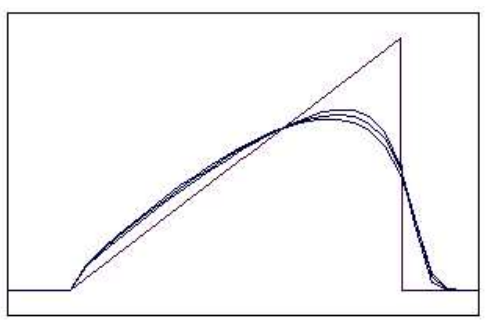

$t=18$

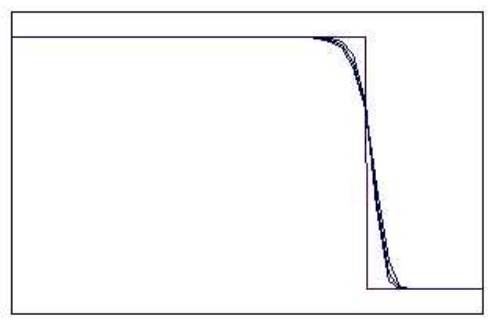

$t=40$

Figure 2. Numerical solution obtained using the implicit upwind scheme for 2 initial data $(\gamma=0.1 ; 0.5 ; 0.9)$.

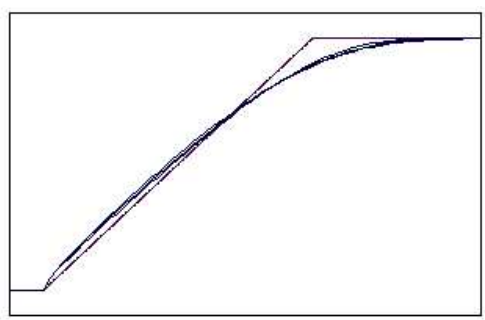

$t=60$

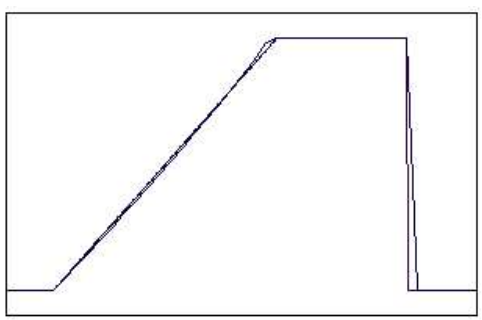

$t=160$

Figure 3. Numerical solution obtained using implicit scheme with upwind difference for 3 initial data $(\gamma=0.1 ; 0.5 ; 0.9)$

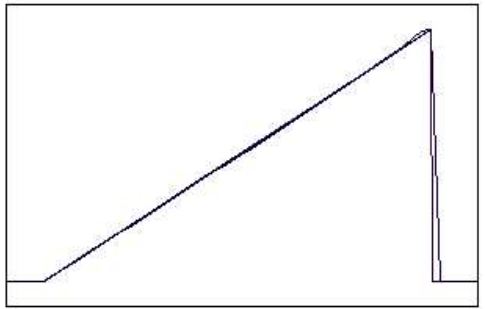

$t=5$

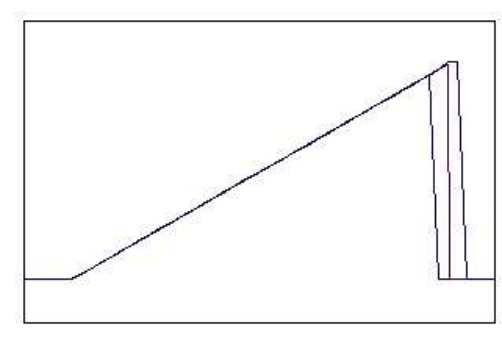

$t=20$

Figure 4. Numerical solution obtained using the implicit upwind scheme for 4 initial data $(\gamma=0.1 ; 0.5 ; 0.9)$ 


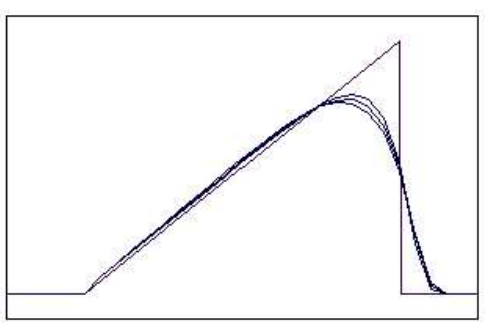

$t=20$



$t=80$

Figure 5. Numerical solution obtained using the implicit upwind scheme for 5 initial data $(\gamma=0.1 ; 0.5 ; 0.9)$

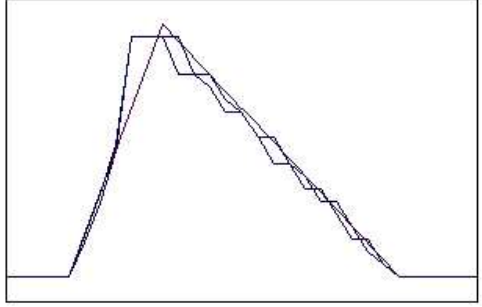

$t=10$

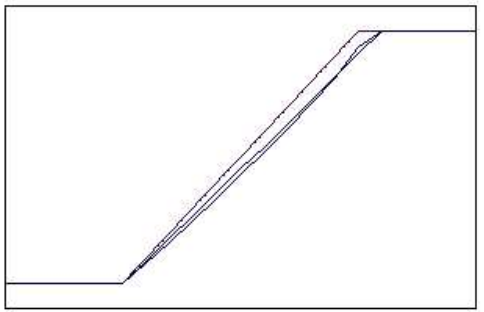

$t=40$

Figure 6. Numerical solution obtained using the implicit upwind scheme for 6 initial data $(\gamma=0.1 ; 0.5 ; 0.9)$

\subsection{The Lax-Wendroff scheme}

The phenomenon of normal dispersion is typical for this scheme. The appearance of shock waves is accompanied by oscillations behind their front. Appearance of oscillations on the left front of generated depression wave is typical for initial profi les 2, 4 and 6 . These oscillations are progressing in time. It leads to growth of errors especially in the $C$ norm. For instance at $\gamma=0.5$ for initial profi le 2 at time moment $t=10$ the relative error in the $C$ norm is equal to 0.17 and at time moment $t=T$ the error is equal to 1.076 .

The spreading of solution is less for the Lax-Wendroff scheme compared with implicit and explicit upwind schemes. Solution discontinuities are reproduced better by this scheme, and discontinuity diffusion takes place on 3 to 5 steps.

\subsection{Jumping advection method}

Compared with schemes given above the numerical solution obtained using the jumping advection method represents the exact solution better. The main advantage of this method is the ability to represent discontinuities such as shock waves 
precisely. But due to features of the method the numerical solution is changing spasmodically and the exact position of shock waves can be obtained at some time moments only. For instance if $\gamma=0.5$ and initial profi le 5 is used, then the solution jumps to the right side by one step for every fourth time layer, overtaking the exact solution. Thus at these moments there is no computational error.

However, when the Courant number increases, defects of numerical solution such as "steps" and wrong speed of shock waves appear. It can be seen in Figure 9 that "steps" appear for the compression wave for initial profi le 4 with any Courant number, and fi gures 8 and 11 show that when $\gamma=0.9$, then shock wave of the numerical solution move slower than the exact one. In Figures 7-11 $\gamma=0.5$ corresponds to the numerical solution which most accurately approximates the exact one.

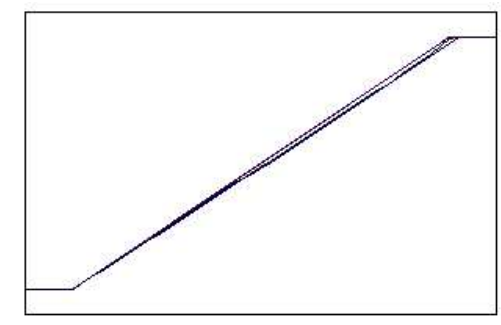

$t=18$

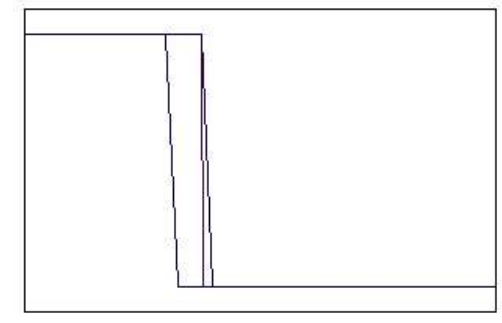

$t=40$

Figure 7. Numerical solution for the jumping advection method for 2 initial data $(\gamma=$ $0.5 ; 0.9)$

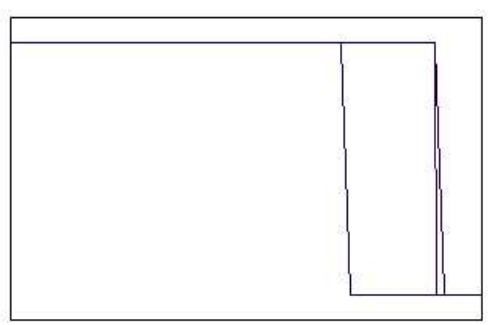

$t=60$

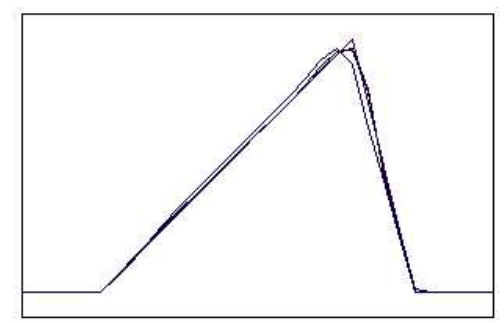

$t=160$

Figure 8. Numerical solution for the jumping advection method for 3 initial data $(\gamma=$ $0.5 ; 0.9)$

\subsection{Monotonized Babenko scheme}

The numerical solution which is obtained using this scheme represents the exact one in the best way compared with schemes examined above in all explored range of Courant numbers. This conclusion follows from the error analysis. This scheme 




$t=5$

Figure 9. Numerical solution for the jumping advection method for 4 initial data $(\gamma=$ $0.5 ; 0.9)$

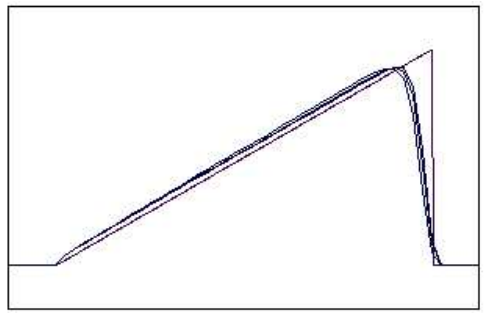

$t=20$

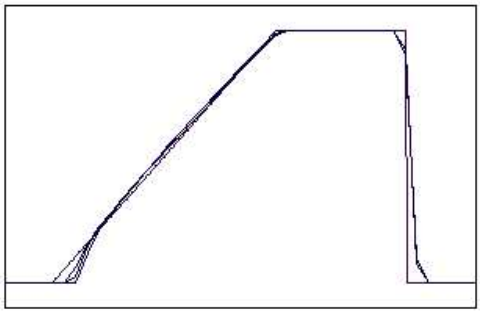

$t=80$

Figure 10. Numerical solution for the jumping advection method for 5 initial data $(\gamma=$ $0.5 ; 0.9)$

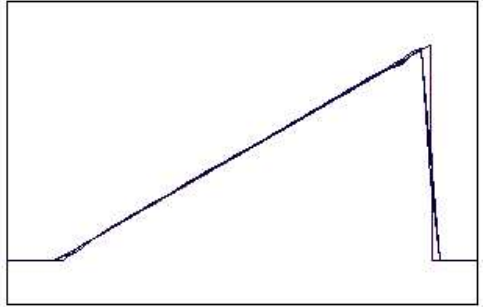

$t=10$

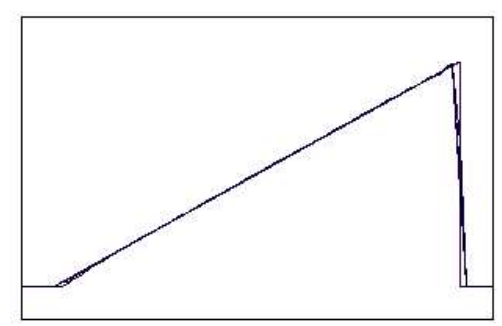

$t=40$

Figure 11. Numerical solution for the jumping advection method for 6 initial data $(\gamma=$ $0.5 ; 0.9)$

has no normal and anomalous dispersion, the solution spreads slightly, discontinuity spreading takes place at smaller number of steps (1-3 steps). For initial profi les 2, 4, 6 little right shifting of a left front of the resulting depression wave can be noticed. As it is shown on Figures 12-17, numerical solutions are almost the same for different Courant numbers. And for all tests $\gamma=0.1$ corresponds to the most accurate numerical solution. 


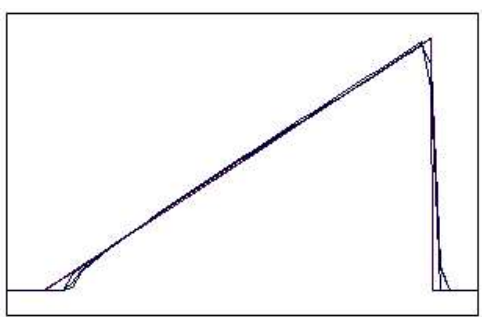

$t=6$

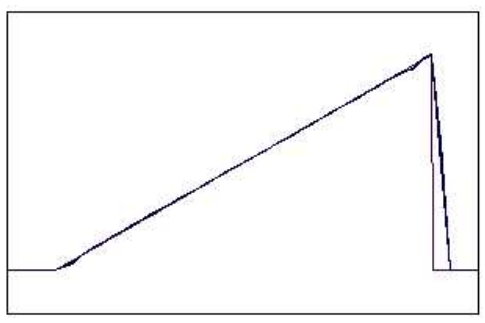

$t=170$

Figure 12. Numerical solution for the Babenko scheme for 1 initial data $(\gamma=0.1 ; 0.5 ; 0.9)$.

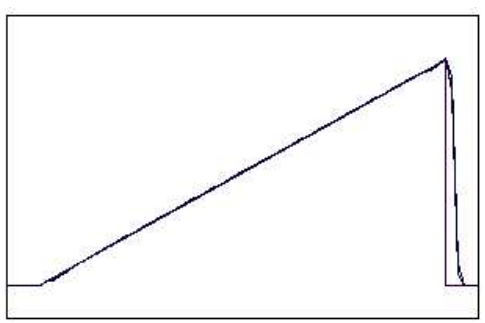

$t=18$

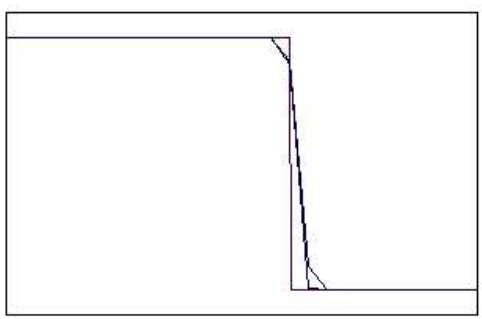

$t=160$

Figure 13. Numerical solution for the Babenko scheme for 2 initial data $(\gamma=0.1 ; 0.5 ; 0.9)$.

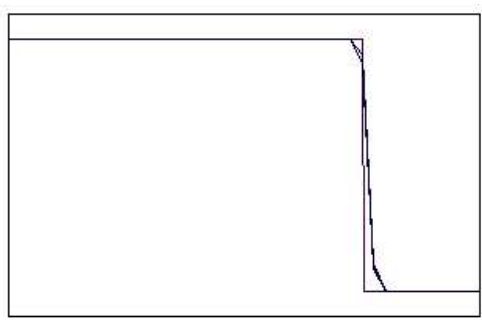

$t=60$

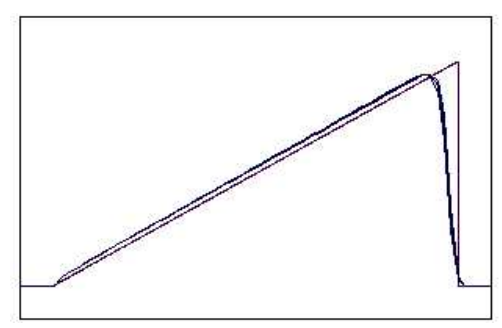

$t=160$

Figure 14. Numerical solution for the Babenko scheme for 3 initial data $(\gamma=0.1 ; 0.5 ; 0.9)$.

\section{Conclusion}

Nonlinear monotonised Babenko scheme, considered in this work, gives the best solution of quasi-linear advection equation in all explored range of Courant numbers. The obvious advantage of this scheme is its ability to present solution discontinuities in a most accurate way compared with other schemes. We notice that the jumping advection method [11] gives worse solution at some initial conditions and for some Courant numbers, despite of its ability to represent the exact solution at some time moments. It is connected to the appearance of "steps" leading to strong solution distortion.

Some additional details can be found in [1]. 


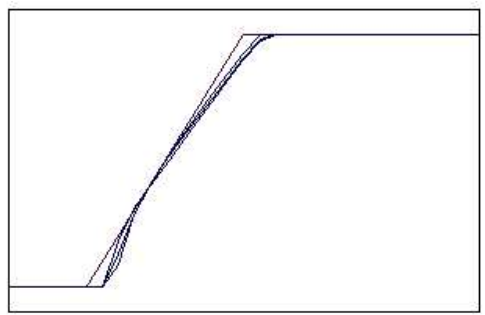

$t=5$

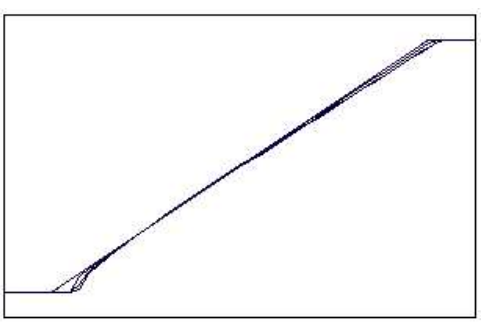

$t=20$

Figure 15. Numerical solution for the Babenko scheme for 4 initial data $(\gamma=0.1 ; 0.5 ; 0.9)$.

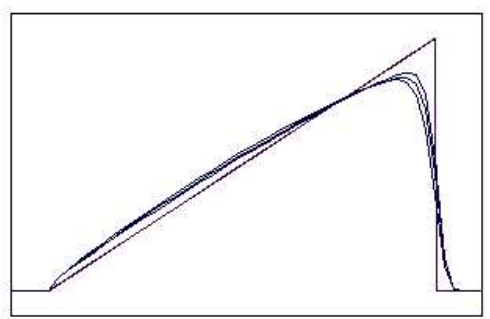

$t=20$

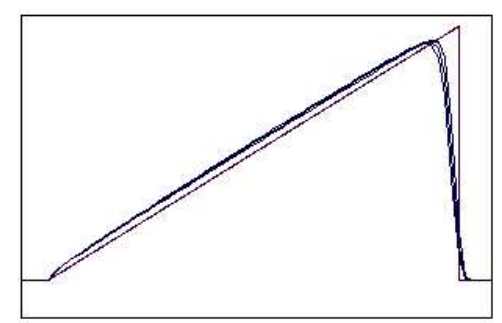

$t=80$

Figure 16. Numerical solution for the Babenko scheme for 5 initial data $(\gamma=0.1 ; 0.5 ; 0.9)$.

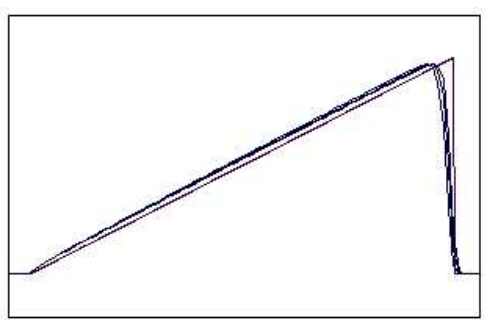

$t=10$

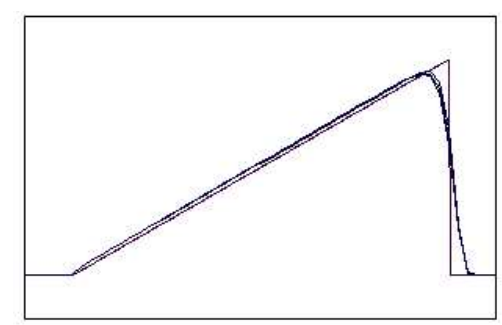

$t=40$

Figure 17. Numerical solution for the Babenko scheme for 6 initial data $(\gamma=0.1 ; 0.5 ; 0.9)$.

\section{References}

[1] T.A. Alexandrikova and M.P. Galanin. Nonlinear monotonization of K.I. Babenko scheme ("square") for the quasi-linear advection equation. Preprint of Keldysh Inst. of Appl. Math. of RAS, 62, 35, 2003. (In Russian)

[2] K.I. Babenko and G.P. Voskresenskii. Numerical method for calculation of a spatial flow over a body by the supersonic gas throughput. J. of Comput. Math. and Math. Phys., 1(6), 1051 - 1060, 1961. (In Russian)

[3] R.P. Fedorenko. Introduction into computational physics. MFTI, Moscow, 1994. (In Russian)

[4] M.P. Galanin and T.G. Yelenina. The comparative analysis of difference schemes for linear advection equation. Preprint of Keldysh Inst. of Appl. Math. of RAS, 52, 33, 1998. (In Russian) 
[5] M.P. Galanin and T.G. Yelenina. The nonlinear monotonization of difference schemes for linear advection equation. Preprint of Keldysh Inst. of Appl. Math. of RAS, 44, 33, 1999. (In Russian)

[6] M.P. Galanin and T.G. Yelenina. The testing of difference schemes for linear advection equation. Preprint of Keldysh Inst. of Appl. Math. of RAS, 40, 42, 1999. (In Russian)

[7] M.P. Galanin and T.G. Yelenina. The finite-difference schemes for solution of linear advection equation. In: Proceedings of the 3rd Int. Conf. Finite-Difference Schemes: Theory and Applications, IMI, Vilnius, 75 - 82, 2000.

[8] M.P. Galanin and T.G. Yelenina. Nonlinear monotonization of K.I. Babenko ("square") scheme for the advection equation. Preprint of Keldysh Inst. of Appl. Math. of RAS, 4, 26, 2002. (In Russian)

[9] M.P. Galanin and T.G. Yelenina. Nonlinear monotonization of the K. I. Babenko scheme ("square"). Mathematical Modelling and Analysis, 8(2), 113 - 120, 2003.

[10] V.M. Goloviznin and S.A. Karabasov. The non-linear correction of "cabaret" scheme. Mathematical Modeling, 12, 107 - 123, 1998. (In Russian)

[11] V.M. Goloviznin and S.A. Karabasov. The jumping advection method for numerical solution of hyperbolic equations. The accurate algorithm for convection simulation on Euler grids. Preprint IBRAE, IBRAE-2000-04, 40, 2002. (In Russian)

[12] A.A. Ivanov, V.F. Tyshkin, A.P. Favorskii and A.N. Yaschook. The construction of quasimonotone schemes of advanced approximation for the advection equation. Preprint of Keldysh Inst. of Appl. Math. of RAS, 69, 25, 1993. (In Russian)

[13] N.N. Kalitkin. Numerical methods. Nauka, Fizmatlit, Moscow, 1978. (In Russian)

[14] B.L. Rogdestvenskii and N.N. Yanenko. Systems of quasi-linear equations. Nauka, Fizmatlit., Moscow, 1978. (In Russian)

[15] A.A. Samarskii. Theory of difference schemes. MFTI, Moscow, 1977. (In Russian)

[16] A.A. Samarskii and Yu.P. Popov. Difference schemes for gas dynamics problems. Nauka, Fizmatlit, Moscow, 1980. (In Russian)

[17] A.N. Tikhonov and A.A. Samarskii. Equations of mathematical physics. Nauka, Fizmatlit, Moscow, 1972. (In Russian)

[18] K.V. Vyasnikov, V.F. Tyshkin and A.P. Favorskii. The construction of monotone difference schemes of advanced approximation for system of equations of hyperbolic type. Matematicheskoye modellirovaniye, 1(5), 95 - 120, 1989. (In Russian)

[19] J. Wizem. Nonlinear waves. Nauka, Fizmatlit., Moscow, 1977. (In Russian)

Babenkos skirtumų schemos kvazi-tiesinei pernešimo lygčiai netiesinè monotonizacija

T.A. Alexandrikova, M.P. Galanin

Šiame straipsnyje pasi-ulyta kvazi-monotonire netiesinė Babenkos skirtumų schema kvazitiesinei pernešimo lygčiai spręsti. Schemos monotoniškumas pasiekiamas pridedant dirbtinę difuziją su apribojimais. Pateiktas šios schemos palyginimas su kitomis schemomis. Taip pat analizuojama antros eilès pagal laiką ir erdvę monotoninè "Cabaret" schema. Pateikti testu rezultatai. 